\title{
Ion Microscopy Goes Quantum
}

\author{
Researchers have developed an ion-optics-based quantum microscope \\ that has sufficient resolution to image individual atoms.
}

\author{
By Arturo Camacho-Guardian
}

( ne of the biggest unsolved problems in condensed-matter physics is explaining the origin of high-temperature superconductivity, a phenomenon where materials conduct electricity with zero resistance at temperatures well above those predicted by theory. Researchers think that the behavior is made possible by strong interactions among a material's constituents. But, because of the complexity of these systems, numerical calculations of their behaviors are unfeasible, making it hard to confirm this-or any other-theoretical idea. Experiments offer opportunities for

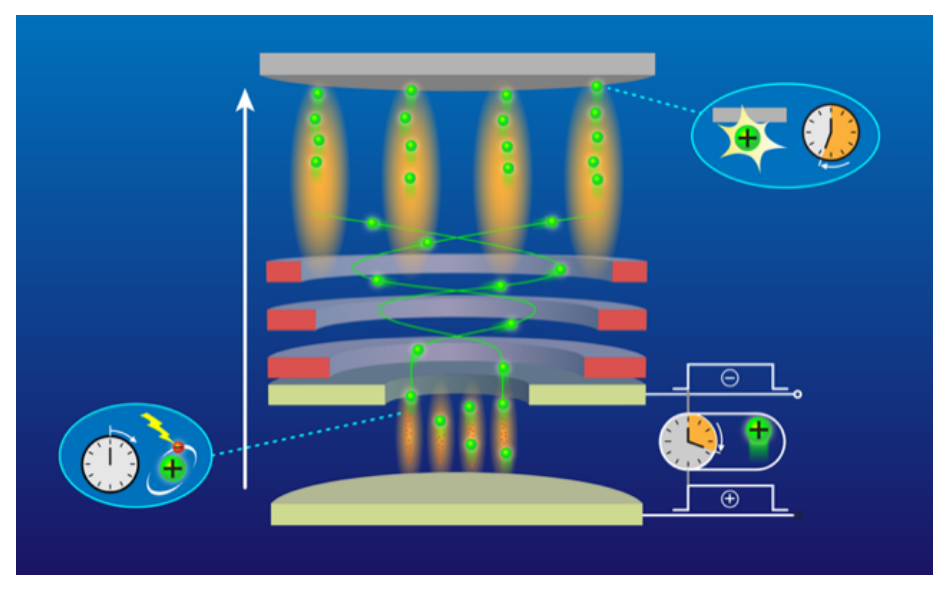

Figure 1: Researchers demonstrate an ion-optics-based microscope that can resolve individual charged atoms. The atoms are confined in a one-dimensional optical lattice (bottom of image) and then illuminated with a light pulse, which ionizes the atoms (green balls). After a short delay, the ionized atoms are transferred into the ion-optic system, where they are manipulated with electrostatic lenses (red rectangles) and imaged with an ion detector (top of image). The arrow indicates the direction of travel of the ions through the microscope.

Credit: APS/Alan Stonebraker tackling the problem, particularly those involving ultracold atomic gases (see Coming Soon: Cold Atoms Impersonate Superconductors). Now a team lead by Tilman Pfau at the University of Stuttgart, Germany, has reported a new tool for researchers studying ultracold gases: a quantum microscope based on ion optics that can resolve individual charged atoms [1]. The technology could provide a means to uncover new insights into strongly interacting materials.

For any microscope to be useful, it must have a resolving power sufficient to image the features of interest. For quantum gases, these features, which include atoms and their spatial order, typically have submicrometer length scales. Such small features are hard to image with traditional-optics-based quantum microscopes, whose highest resolution is $5 \mu \mathrm{m}$.

This resolution problem was partially solved in 2009 with the arrival of the first quantum gas microscope (QGM), which can capture features down to $0.5 \mu \mathrm{m}$ in size [2]. The device allows researchers to arrange atoms in a lattice pattern and then image the atoms using the fluorescent light they emit.

QGMs are used to image arrays of fermions (in this case, atoms with an odd number of neutrons) to uncover behaviors that mimic those of electrons in solid materials. Although QGMs haven't elucidated the high-temperature-superconducting mechanism yet, researchers are hopeful that will happen soon. But the QGM has an important limitation: It cannot image electronically excited atoms, such as Rydberg atoms, which can form exotic phases, including quantum crystals.

The problem lies with the microscope's use of fluorescent light. Fluorescence imaging relies on a closed-cycle transition, where an atom absorbs light, moving from one state to another, and then spontaneously emits light and decays back to its initial 
state. These closed cycles exist for ground-state atoms, such as the fermions imaged with QGM, but they can't always be found for excited atoms. To image excited atoms, researchers need to leverage other phenomena, which is what Pfau and his colleagues have done.

The team designed an ion-optics-based system. Their microscope consists of three electrostatic lenses and an ion detector that can count single ions (Fig. 1). Electrostatic lenses work using the same principles as other lenses, such as those in cameras and telescopes, which focus light using transparent curved surfaces made of glass or plastic. But, rather than manipulating light with an object, electrostatic lenses manipulate the paths of ions using electric fields. The two sets of lenses also have another key difference: The magnification of a given traditional lens is fixed, while that of an electrostatic lens is adjustable. This adjustment is made by changing the voltage of the electric fields used to create the lens, something Pfau and his colleagues leveraged in their experiments.

In the microscope, an ultracold gas of neutral atoms is first confined in a one-dimensional lattice, as in a QGM. (Pfau and colleagues used rubidium atoms for their demonstration.) The atoms are then illuminated with a series of laser pulses. Each pulse charges between 10 and 100 atoms in a process known as photoionization. The resulting ions remain in their lattice for between $30 \mathrm{~ns}$ and $4 \mu \mathrm{s}$, enough time for them to interact and build up the many-body correlations that researchers are interested in. The ions are then released into the ion microscope where they are imaged.

The images collected by the team show that their microscope can capture features from $6.79 \mu \mathrm{m}$ down to $0.52 \mu \mathrm{m}$ in size, sufficient to image individual atoms. This maximum resolution is more than 5 times better than that achieved in 2017 with a different ion-optics-based microscope, which had a resolution of $2.7 \mu \mathrm{m}$ [3]. The new microscope also has a depth of field of $70 \mu \mathrm{m}$. This depth is an order of magnitude larger than that of a QGM, which has a depth of field of only a few micrometers and is a big enough distance to potentially use the microscope to capture 3D images.

This increased resolution could allow the new microscope to explore previously uncharted territory for excited atoms. For example, the microscope could be used as a tool for monitoring the so-called dressing of an impurity by its environment, a process that modifies the impurity's properties, such as energy, charge, and mass. Dressed impurities embedded in a strongly interacting bath are predicted to play a crucial role in the behavior of high-temperature superconductors.

Ion-optics-based microscopy also raises the possibility of unveiling the interplay between few-body correlations and macroscopic phases of matter $[4,5]$. If the microscope can achieve a high-time resolution, it could help reveal the crossover between short-time dynamics, where the system is governed by two-body collisions, and long-time dynamics, where many-body correlations can lead to the formation of intriguing new quantum states. So far, this crossover has only been observed for ground-state atoms $[6,7]$.

Finally, the single-site resolution achieved by Pfau and colleagues could allow for the study of few- and many-body effects, such as crystallization, in gases containing Rydberg atoms [8] as well as in quantum phases prepared from ground-state atoms. These studies could provide a deeper understanding of the most complex strongly correlated systems in atomic and condensed-matter physics.

Arturo Camacho-Guardian: University of Cambridge, Cambridge, United Kingdom

\section{REFERENCES}

1. C. Veit et al., "Pulsed ion microscope to probe quantum gases," Phys. Rev. X 11, 011036 (2021).

2. W. S. Bakr et al., "A quantum gas microscope for detecting single atoms in a Hubbard-regime optical lattice," Nature 462, 74 (2009).

3. M. Stecker et al., "A high resolution ion microscope for cold atoms," New J. Phys. 19, 043020 (2017).

4. G. E. Astrakharchik et al., "Ionic polaron in a Bose-Einstein condensate," arXiv:2005.12033.

5. E. R. Christensen et al., "Charged polarons and molecules in a Bose-Einstein condensate," arXiv:2012.11436.

6. M. Cetina et al., "Ultrafast many-body interferometry of impurities coupled to a Fermi sea," Science 354, 96 (2016).

7. M. Skou et al., "Non-equilibrium dynamics of quantum impurities," arXiv:2005.00424.

8. P. Schauss et al., "Crystallization in Ising quantum magnets," Science 347, 1455 (2015). 